\title{
Recent insights into the biological activities and drug delivery systems of tanshinones
}

REVIEW

\author{
This article was published in the following Dove Press journal: \\ International Journal of Nanomedicine \\ 5 January 2016 \\ Number of times this article has been viewed
}

\author{
Yuee Cai ${ }^{1}, *$ \\ Wenji Zhang',* \\ Zirong Chen ${ }^{3}$ \\ Zhi Shi ${ }^{1,2}$ \\ Chengwei $\mathrm{He}^{1}$ \\ Meiwan Chen' \\ 'State Key Laboratory of Quality \\ Research in Chinese Medicine, \\ Institute of Chinese Medical Sciences, \\ University of Macau, Macau, People's \\ Republic of China; ${ }^{2}$ Department of Cell \\ Biology \& Institute of Biomedicine, \\ National Engineering Research Center \\ of Genetic Medicine, Guangdong \\ Provincial Key Laboratory of \\ Bioengineering Medicine, College of Life \\ Science and Technology, Jinan University, \\ Guangzhou, Guangdong, People's \\ Republic of China; ${ }^{3}$ Department of \\ Molecular Genetics and Microbiology, \\ Shands Cancer Center, University of \\ Florida, Gainesville, FL, USA \\ *These authors contributed equally \\ to this work
}

Correspondence: Meiwan Chen State Key Laboratory of Quality Research in Chinese Medicine, Institute of Chinese Medical Sciences, University of Macau, Avenida da Universidade, Taipa, Macau, People's Republic of China

Tel +853 88224675

Fax +8532884 I358

Email mwchen@umac.mo

Zhi Shi

Department of Cell Biology \& Institute of Biomedicine, National Engineering Research Center of Genetic Medicine, Guangdong Provincial Key Laboratory of Bioengineering Medicine, College of Life Science and Technology, Jinan University, 60I Huangpu Avenue West, Guangzhou 510632, People's Republic of China

Tel +862085224525

Fax +86 2085225977

Email tshizhi@jnu.edu.cn

\begin{abstract}
Tanshinones, the major lipid-soluble pharmacological constituents of the Chinese medicinal herb Tanshen (Salvia miltiorrhiza), have attracted growing scientific attention because of the prospective biomedical applications of these compounds. Numerous pharmacological activities, including anti-inflammatory, anticancer, and cardio-cerebrovascular protection activities, are exhibited by the three primary bioactive constituents among the tanshinones, ie, tanshinone I (TNI), tanshinone IIA (TNIIA), and cryptotanshinone (CPT). However, due to their poor solubility and low dissolution rate, the clinical applications of TNI, TNIIA, and CPT are limited. To solve these problems, many studies have focused on loading tanshinones into liposomes, nanoparticles, microemulsions, cyclodextrin inclusions, solid dispersions, and so on. In this review, we aim to offer an updated summary of the biological activities and drug delivery systems of tanshinones to provide a reference for these constituents in clinical applications.
\end{abstract}

Keywords: tanshinones, biological activities, drug delivery systems, bioavailability, solubility

\section{Introduction}

Tanshen, also known as Salvia miltiorrhiza, red sage, Chinese sage, or danshen, is widely used in traditional Chinese medicine for the treatment of cerebrovascular and cardiovascular diseases, as well as inflammatory diseases. ${ }^{1}$ Tanshinones are the major liposoluble constituents of Tanshen and consist of abietane type-diterpene quinone pigments. $^{2}$ The primary bioactive constituents among the tanshinones are tanshinone I (TNI), tanshinone IIA (TNIIA), and cryptotanshinone (CPT) (Figure 1), which have attracted special attention for possessing various pharmacological effects, including anti-inflammatory, anticancer, and cardio-cerebrovascular protection activities. ${ }^{3,4}$ TNIIA, which has been studied from the 1930s, has potential effects against diabetes, neurodegenerative diseases, and cardiac hypertrophy. ${ }^{5}$ In addition to the anti-inflammatory and anticancer effects, TNI also enhances the ability to memorize and learn and ameliorates memory impairment. ${ }^{6-8}$ Recently, a number of studies have been published concerning the modification, biosynthesis, metabolism, pharmacological actions, and therapeutic applications of tanshinones. ${ }^{5,9-15}$ However, the poor water solubility and low oral bioavailability of tanshinones have limited their clinical applications. There have been no reviews that focused on the drug delivery systems of tanshinones to date. To give summary of current research progress of tanshinones, the organization of this review is as described later. First of all, the review outlines the various pharmacological of tanshinones (Figure 2), which indicates the high medicinal value of them. Then, the development of drug delivery systems for tanshinones are discussed, including liposomes, solid dispersion, and nanoparticles (NPs), which has been used to solve the poor bioavailability of tanshinones. Finally, we make a conclusion of our personal perspectives on the directions for developing tanshinone. 


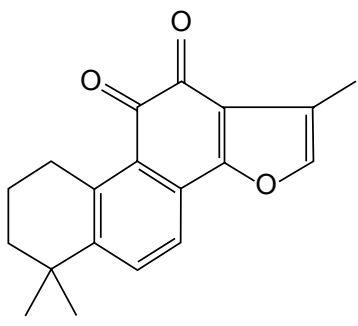

Tanshinone IIA<smiles>Cc1coc2c1C(=O)C(=O)c1c-2ccc2c(C)cccc12</smiles>

Tanshinone I<smiles>CC1COC2=C1C(=O)C(=O)c1c2ccc2c1CCCC2(C)C</smiles>

Cryptotanshinone

Figure I Chemical structures of major tanshinones.

\section{Biological activities}

\section{Cardio-cerebrovascular protection}

Tanshinones have been investigated for the treatment of cardio-cerebrovascular diseases, such as myocardial infarction, atherosclerosis, hyperlipidemia, hypertension, and stroke. ${ }^{4,10,16}$ TNIIA and its derivative, sodium TNIIA sulfonate (STS), were found to significantly reduce the size of a myocardial infarct and improve cardiac function via the activation of the phosphatidylinositol 3 kinase $(\mathrm{PI} 3 \mathrm{~K}) /$ protein kinase B (Akt) signaling pathway in the myocardium..$^{10,17-19}$ TNIIA also inhibited the formation of atherosclerotic lesions and hyperlipidemia by reducing the oxidation of low-density lipoproteins, cholesterol accumulation in macrophages, platelet aggregation, and monocyte adhesion to the endothelium..$^{10,20-22}$ In addition, TNIIA and STS attenuated pulmonary hypertension by modulating calcium and potassium channels and inhibited the proliferation and migration of vascular smooth muscle cells by blocking the Akt pathway. ${ }^{23-26}$ TNIIA and TNI protect the brain from ischemic damage in stroke models by reducing the brain infarct volume and restoring neurological function, which might be correlated with the induced nuclear translocation of transducer of regulated cAMP response element-binding protein (CREB) 1 (TORC1) and upregulated expression of phosphorylated (pCREB), TORC1, and brain-derived

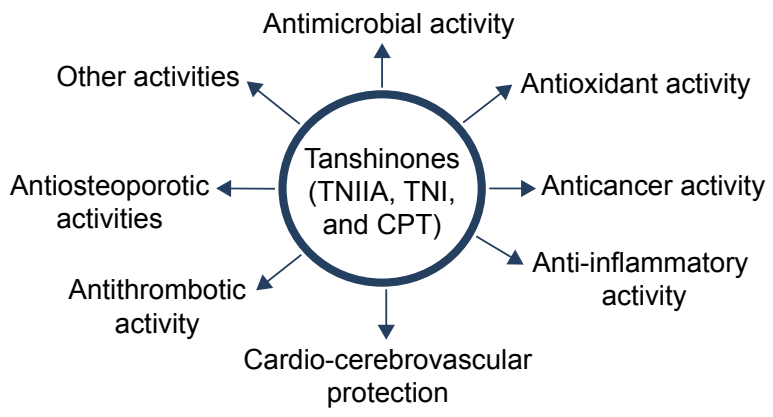

Figure 2 Biological activities of TNIIA, TNI, and CPT.

Abbreviations: TNIIA, tanshinone IIA; TNI, tanshinone I; CPT, cryptotanshinone. neurotrophic factor. ${ }^{27-29}$ In a clinic study of 100 unstable angina pectoris (UAP) patients, $60 \mathrm{mg}$ STS in combination with $300 \mathrm{mg}$ aspirin can significantly attenuate angina pectoris attacks. ${ }^{30}$ The clinical trials of S. miltiorrhiza and tanshinones in patients with ischemic conditions have been well summarized in other reviews. ${ }^{1,31}$

\section{Anticancer activities}

Recently, the anticancer activities of tanshinones have been systematically summarized in an informative review. ${ }^{15}$ TNIIA, TNI, and CPT are potent cytotoxic agents that significantly inhibit the growth and survival of multiple types of cancer cells by inducing cell cycle arrest and apoptosis with $\mathrm{IC}_{50}$ at the micromolar level. The potential mechanisms involved include the upregulation of pro-apoptosis proteins such as p53, Bax, p21, etc, downregulation of antiapoptosis proteins, including Bcl-2, survivin, and c-Myc and activation of caspase proteins to trigger cell apoptosis. ${ }^{15,32}$ TNIIA was also found to induce autophagic cell death in various cancer cells by activating AMP-activated protein kinase and extracellular signal-regulated kinase (ERK) and inhibiting the mammalian target of rapamycin and $70 \mathrm{kDa}$ ribosomal protein $\mathrm{S} 6$ kinase signaling pathways. ${ }^{33}$ In addition, TNIIA and TNI are able to inhibit the migration, invasion, and metastasis of cancer cells through the alteration of matrix metalloproteinases and/or tissue inhibitor of metalloproteinases..$^{34-38}$ Notably, TNIIA can also promote cell differentiation in several cancer cell types likely by regulating CCAAT/enhancer binding protein $(\mathrm{C} / \mathrm{EBP})$ beta and C/EBP homologous protein $10{ }^{39-41}$ Furthermore, TNIIA, TNI, and CPT exhibited promising anticancer effects with minor side effects in many xenograft animal models. ${ }^{15,36,38,42,43}$ TNIIA, TNI, and CPT have synergistic anticancer effects with chemotherapeutic drugs such as cisplatin, doxorubicin, 5-flurouracid, and arsenic trioxide. ${ }^{44-48}$ Moreover, TNIIA, TNI, and CPT can overcome P-glycoprotein (P-gp)-mediated multidrug resistance in cancer by acting as substrates of 
P-gp and suppressing its pump activity. ${ }^{48-51}$ The clinical use of TIIA for cancer treatment has been reported in a patient with acute promyelocytic leukemia, who had no response to all-trans retinoic acid (ATRA) (20 mg, three times per day) for 14 days, and they achieved a complete remission after treated with TIIA (30 mg, oral, two times per day) for 12 weeks. ${ }^{52}$ Cancer-related clinical trials of TIIA and tanshinone-containing traditional Chinese medicine formulas were well presented in this review. ${ }^{53}$

\section{Anti-inflammatory activities}

TNIIA has shown remarkable anti-inflammatory activity by inhibiting the expression of inflammatory mediators, including interleukin (IL)-1 beta, IL-6, and tumor necrosis factor (TNF)-alpha, in an estrogen receptor subtype-dependent manner in murine macrophage RAW264.7 cells pretreated with lipopolysaccharide (LPS). ${ }^{54}$ Additionally, TNIIA suppresses LPS-induced nuclear factor kappaB $(\mathrm{NF}-\kappa \mathrm{B})$ activation through the inhibition of the NF- $\mathrm{KB}$-inducing kinase/ IkappaB alpha kinase (NIK/IKKalpha), ERK1/2, p38, and c-Jun N-terminal kinase (JNK) pathways. ${ }^{55}$ Recently, data from a protein interaction network analysis have suggested that the anti-inflammatory effect of TNIIA may result in part from activating TNF receptor-associated factor (TRAF) $2 / 3 / 6$ and inhibiting the toll-like receptor (TLR) signaling pathway. ${ }^{56}$ TNI significantly inhibited the activity of group IIA secretory phospholipase $A_{2}$ (GIIA) to thereby block prostaglandin $\mathrm{E}_{2}$ (PGE2) formation in LPS-activated macrophages and exhibited in vivo anti-inflammatory activity in rats with adjuvant-induced arthritis and carrageenan-induced paw edema. ${ }^{57}$ TNI and CPT also significantly inhibit IL-12 production in LPS-activated macrophages and interferon- $\gamma$ production in lymph node cells. ${ }^{58}$

\section{Other pharmacological activities}

Tanshinones are known as natural antioxidants by forming a quinone adduct of the lipid radical to form a stabilized radical. ${ }^{2}$ TNIIA is able to prevent the DNA damage in liver cells resulting from lipid peroxidation by scavenging lipid free radicals and breaking the peroxidation chain reactions. ${ }^{59}$ Preincubation with TNIIA significantly decreases the $\mathrm{H}_{2} \mathrm{O}_{2}$ induced death of ECV-304 human umbilical vein endothelial cells and $\mathbf{J 7 7 4}$ macrophages. ${ }^{60,61}$ In addition, TNI activated the NF-E2 p45-related factor 2 (Nrf2)-dependent antioxidant response by preventing ubiquitination-mediated Nrf2 degradation and protected against As (III)-induced lung damage in vitro and in vivo. ${ }^{62}$ TNI inhibited peroxynitrite-induced DNA damage by diminishing the 5,5-dimethyl-1-pyrroline
$N$-oxide-hydroxyl (DMPO-OH) radical adduct signal from peroxynitrite. ${ }^{63}$ Tanshinones also have potent antiosteoporotic activities by targeting different pathways in the bone remodeling cycle. ${ }^{64}$ It has been demonstrated that TNIIA, TNI, and CPT have obvious inhibitory effects on osteoclast differentiation. ${ }^{65}$ It was found in further experiments that TNIIA inhibits osteoclast differentiation through blocking Akt, ERK, and NF- $\mathrm{KB}$ activation and downregulating the expression of c-Fos and nuclear factor of activated T-cells (NFATC1), cytoplasmic, calcineurin-dependent 1, which all are induced by receptor activator of NF- $\mathrm{kB}$ ligand. ${ }^{66,67}$ Furthermore, TNIIA also suppressed bone resorption of differentiated osteoclasts. ${ }^{67}$ Notably, TNIIA and TNI have insulin-sensitizing effects and enhance the ability of insulin to promote the tyrosine phosphorylation of the insulin receptor and the activation of the downstream ERK, Akt, and glycogen synthase kinase-3 beta (GSK-3 3 ) kinases in Chinese hamster ovary cells and 3T3-L1 adipocytes. ${ }^{68}$ It has been shown that CPT has potent antibacterial activity against a wide range of Gram-positive bacteria in a reactive oxygen species (ROS)-dependent manner ${ }^{69}$ and has the potential to treat Alzheimer's disease as an inhibitor of acetylcholinesterase. ${ }^{70}$ Particularly, major tanshinones such as TNIIA, TNI, and CPT are able to competitively inhibit the metabolism of CYP1A2 substrates, ${ }^{71}$ and TNIIA and CPT are efficacious pregnane $\mathrm{X}$ receptor (PXR) agonists that induce CYP3A4 expression, suggesting that attention should be paid when tanshinones are used in combination with drugs metabolized by CYP1A2 and CYP3A4. ${ }^{72}$ Recently, TNIIA, TNI, and CPT were found to be specific and selective inhibitors for the SARS-CoV cysteine proteases $3 \mathrm{CL}^{\text {pro }}$ and $\mathrm{PL}^{\text {pro }}$, but did not exert significant inhibitory effects against other proteases, including chymotrypsin, papain, and HIV protease. ${ }^{73}$ Moreover, TNIIA could inhibit tat-induced HIV-1 transactivation through redox-regulated AMPK/NAMPT pathway. ${ }^{74}$

\section{Drug delivery systems}

Despite the multiple pharmacological effects of TNIIA, the poor water-solubility and low dissolution rate of this compound result in low oral bioavailability and have hampered the clinical application of TNIIA. ${ }^{75}$ To tackle this problem, various methods have been developed, including the preparation of STS, the water-soluble derivative of TNIIA, the preparation of TNIIA in discoidal and spherical high-density lipoproteins, and the development of drug delivery systems for TNIIA. ${ }^{5}$ To date, many studies have focused on loading TNIIA into liposomes or NPs, microemulsions, cyclodextrin (CD) inclusion, and solid dispersions. ${ }^{76-80}$ In addition, 
preparations of TNI and CPT with better intestinal absorption have been studied in recent years due to the unsatisfactory clinical effects of these agents, which have been attributed to their low levels of biological utilization; ${ }^{81,82}$ these preparations include solid dispersion and solid lipid NPs. ${ }^{81,83}$ Table 1 shows the various drug delivery systems of tanshinones.

\section{Liposomes}

Liposomes are closed spherical vesicles composed of a lipid bilayer and have attractive properties, including biocompatibility, biodegradability, low clearance rates, and low toxicity. ${ }^{84}$ To establish a highly efficient delivery system with multiple functions, more than one therapeutic drug can be entrapped within the aqueous liposomal interior or embedded into the liposomal membrane, depending on the characteristics of the drug and the process of encapsulation. ${ }^{85}$ Because of these benefits, liposomes were used to encapsulate glycyrrhetinic acid (GA), salvianolic acid B (SB), and TNIIA, enhancing the bioavailability and water solubility of the compounds, which then exerted synergistic effects on the inhibition of hepatic stellate cell (HSC) proliferation. ${ }^{76}$ In this study, TSIIA and GA, the hydrophobic constituents, were incorporated into phospholipid bilayers by employing the film hydration method with probe sonication, and the $\mathrm{pH}$-gradient method was then used to load the hydrophilic constituent SB, which finally yielded the GA-TNIIA-SB compound liposomes (GTS-lip) with cholesterol and soybean phospholipids. Eventually, the encapsulation efficiency of these drugs was $>80 \%$ with little difference among the individual compounds. This study demonstrated that GTS-lip could suppress the proliferation of HSC with a sustainedrelease effect more effectively than a mixed solution of GA, TNIIA, and SB, which may promote the clinical application and therapeutic activities of tanshinones. ${ }^{76}$ The outstanding advantage of the liposomal drug delivery system is the ability to co-encapsulate different drugs to exert synergistic effects in a sustained-release manner, which is more efficient than treatment with unencapsulated drugs. However, a challenge still exists in controlling the ratio of different drugs to produce an improved healing efficacy.

\section{Emulsions}

Emulsions are a class of formulation consisting of two immiscible phases stabilized by a surfactant ${ }^{86}$ and are widely used to enhance the stability of active constituents, thereby maintaining their effectiveness. ${ }^{87}$ In previous studies, lipid emulsions, ${ }^{88}$ microemulsions,${ }^{89}$ and nanoemulsions ${ }^{90}$ of soybean phospholipid, pluronic F68 (F68), glycerol, oleic acid, lecithin, and so on were used to produce TNIIA formulations with long-term stability and obvious anticancer activity.

However, nanoemulsions have proved to have higher bioaccessibility, stability, and optical clarity than conventional emulsions; ${ }^{91,92}$ nanoemulsions are similar to microemulsions that are monodispersed spherical droplets with thermodynamic stability as well as excellent solubility properties. ${ }^{93,94}$ In recent years, a series of studies have been reported regarding the preparation processes, quality control, and evaluation profiles of tanshinone microemulsions. ${ }^{89,90,95-97}$ TNIIA was encapsulated into a microemulsion, consisting of F68, phospholipid, ethyl oleate, and glycerol, which had an antitumor effect on hepatoma H22 cells and mice. ${ }^{89}$ Moreover, another TNIIA microemulsion with a mean droplet size of $32.25 \pm 6.59 \mathrm{~nm}$ was prepared to improve the bioavailability in rat small intestine. The results of the absorption in small intestine of TNIIA microemulsion showed that TNIIA microemulsion could improve the absorption of TNIIA in rat small intestine with the influence of the water-phase ratio (TNIIA microemulsion) to the absorption coefficient. ${ }^{97}$ Additionally, TNIIA nanoemulsions were formed with an average particle size of $95.6 \mathrm{~nm}$ and an excellent entrapment efficiency of $99.3 \%$, which showed potent cytotoxicity with 103.4-fold greater than TNIIA alone against T24 human bladder cancer cells in a time- and dose-dependent manner. ${ }^{90} \mathrm{It}$ was also reported that a tanshinone microemulsion possessed cytotoxic effects on the human leukemia cell line K562/ADM, ${ }^{95}$ and a method of establishing the high performance liquid chromatography fingerprints of tanshinone microemulsions has been developed to control the quality. ${ }^{96}$

Although TNIIA emulsions have excellent long-term stability and anticancer activity, it is not convenient enough to prepare the emulsions with more than four types of materials, which may hinder the application of TNIIA emulsions with unsatisfactory bioavailability.

\section{$C D$ inclusion}

$\mathrm{CD}$ and its derivatives, including $\alpha-\mathrm{CD}, \beta-\mathrm{CD}, \gamma-\mathrm{CD}$, and 2-hydroxypropyl-beta-cyclodextrin (HP- $\beta$-CD), have a cagelike supramolecular structure and the ability to form inclusion complexes with various molecules; these complexes have been used as drug carriers in a number of applications, such as nasal administration, oral drug delivery, and dermal drug delivery, to enhance the stability, solubility, and bioavailability of the drugs. ${ }^{98}$ Hence, the inclusion complexes of TNIIA or TNI and CDs were obtained via coprecipitation and lyophilization to enhance the level of biological utilization, and the results proved that HP- $\beta-C D$ had a greater stability than $\beta$-CD..${ }^{99}$ Furthermore, the transport mechanism 


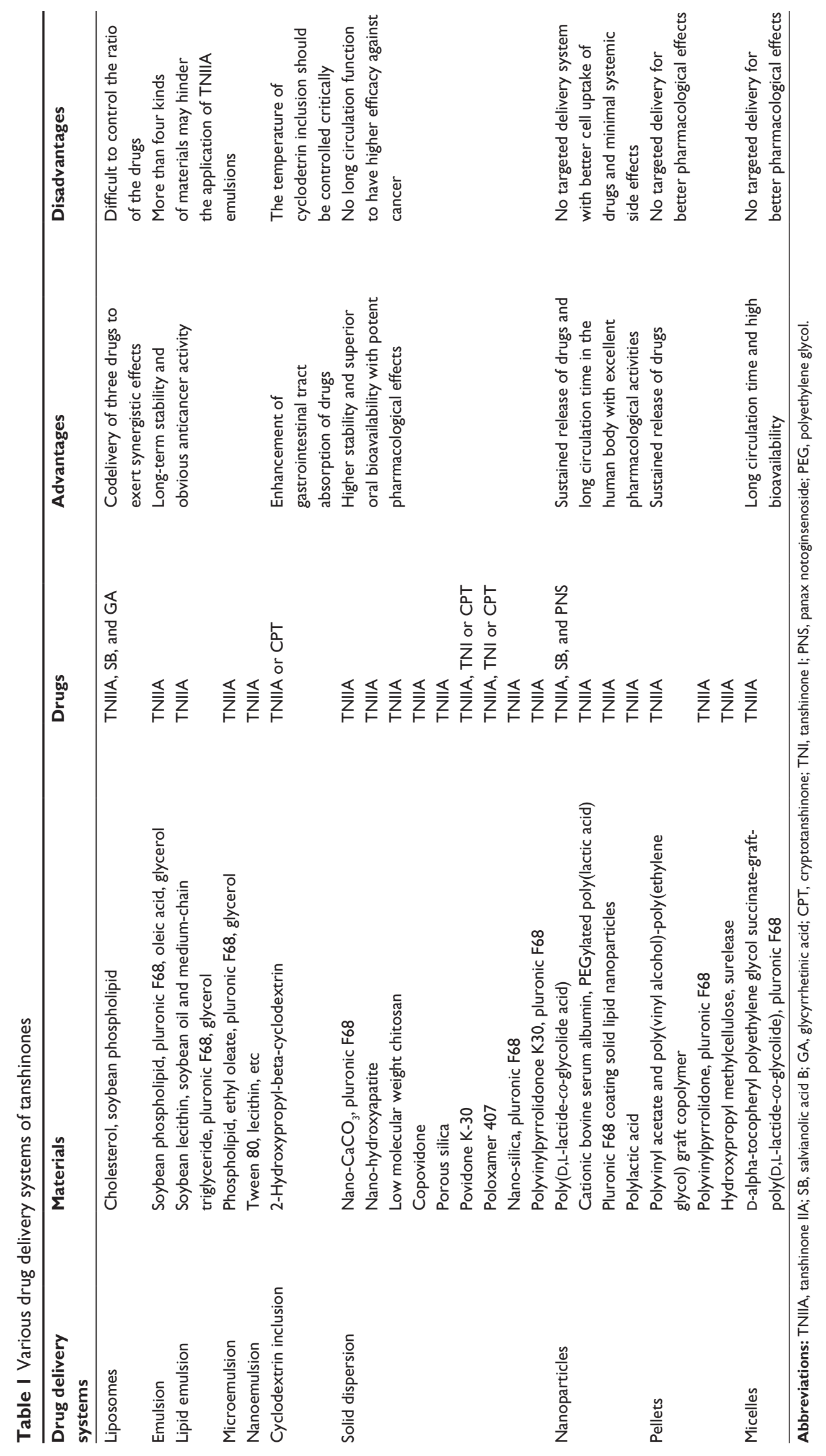


of TNIIA-HP- $\beta$-CD was found by the recirculation intestinal perfusion technique ${ }^{100}$ to be passive transport with no particular absorption site for TNIIA in vivo, and the permeability rate of TNIIA-HP- $\beta$-CD through the intestinal epithelial membrane was seven times higher than that of free TNIIA, indicating that the TNIIA-HP- $\beta$-CD complex enhanced the gastrointestinal tract absorption of TNIIA. ${ }^{77}$ In addition, inclusion complexes of CPT with HP- $\beta$-CD (inclusion ratio $1: 1)$ were prepared by the wet grinding method, which significantly improved the dissolution of CPT. ${ }^{101}$

\section{Solid dispersions}

The production of solid dispersions is a technique for improving the dissolution rate and bioavailability of poorly water-soluble drugs by dispersing the drugs into solidstate hydrophilic carriers with an increased surface area. ${ }^{102}$ Therefore, solid dispersions of TNIIA, TNI, and CPT have been applied to improve the dissolution, stability, and bioavailability of these tanshinones. There are various types of carriers being used to prepare solid dispersions of TNIIA via the solvent technique or spray-drying method, such as combinations of polyvinylpyrrolidone (PVP)-K30 and F68, ${ }^{103}$ nano-silica and $\mathrm{F} 68,{ }^{104}$ poloxamer 407 or povidone $\mathrm{K}-30,{ }^{81}$ porous silica, ${ }^{105}$ copovidone (PVP-S630), ${ }^{106}$ low molecular weight chitosan (LMC), ${ }^{17}$ nano- $\mathrm{CaCO}_{3}$, and F68. ${ }^{80}$ Among TNIIA-poloxamer407, TNIIA-HP- $\beta-C D$, and TNIIA-PVP$\mathrm{K} 30$, the best dissolution rate of TNIIA solid dispersions was TNIIA-poloxamer407. ${ }^{81}$ In addition, TNIIA-PVP-S630 had better dissolution rates than TNIIA-silica NPs and TNIIAnano-silica-F68. ${ }^{104-106}$ Moreover, TNIIA-PVP-S630 at a proportion of 1:10 improved the solubility up to $100 \%$ at 30 minutes and could be stored for 3 months with no change in the dissolution or components and little moisture absorption, which was better than TNIIA-poloxamer407. ${ }^{106}$ Moreover, LMC and TNIIA (weight ratio 9:1) were used to prepare a solid dispersion that increased the dissolution rate by $368.2 \%$ compared to free TNIIA, which could enhance the absorption rate and oral bioavailability. ${ }^{17}$ It was also reported that ternary solid dispersions that were composed of $\mathrm{F} 68$, nano- $\mathrm{CaCO}_{3}$, and TNIIA demonstrated high dissolution rates and stability, representing a promising method to prepare solid dispersions. ${ }^{80}$ Despite the benefits of solid dispersions mentioned earlier, it is necessary to obtain long-lived circulation within the human body and greater efficacy against cancer.

\section{Nanoparticles}

NPs, loosely defined as particles with 1-100 nm diameters, ${ }^{107}$ can enhance the solubility and dissolution rate of drugs by decreasing the particle size and enlarging the surface area. ${ }^{105}$ Therefore, a number of polymeric carriers have been used to prepare NPs of TNIIA with better bioavailability, such as poly(D,L-lactic-co-glycolic acid) (PLGA), ${ }^{108,109}$ polylactic acid (PLA), ${ }^{110} \mathrm{~F} 68,{ }^{111}$ and cationic bovine serum albumin (CBSA)-conjugated PEGylated PLA. ${ }^{78}$ NPs of TNIIA-loaded PLGA (TNIIA-PLGA-NPs) had good biocompatibility and high absorption with preventive effects in the neointimal hyperplasia of the rabbit carotid artery after intimal denudation. ${ }^{109}$ Furthermore, it is more efficient to prepare TNIIA-PLGA-NPs by the nanoprecipitation method instead of the emulsion evaporation method, resulting in particles with average diameters of $225 \mathrm{~nm}$, an entrapment efficiency of $95.49 \%$, a drug loading of $2.03 \%$, and drug recovery rate of $38.42 \% .{ }^{108}$ Another type of material, PLA, was used to prepare novel NPs of TNIIA (TNIIA-PLA-NPs) by a single oil-in-water emulsion/solvent evaporation method, and the resulting particles had a significant dose- and time-dependent growth-inhibitory effect on hepatomas with sustained release in vitro and in vivo, which had 2.69-fold survival time of mice bearing hepatoma tumor than TNIIA. ${ }^{110}$ Despite the enhanced bioavailability and water solubility of TNIIA with the NPs mentioned earlier, it is more efficient to modify the NPs to obtain an extended circulation within the human body. As a result, TNIIA NPs were modified with PEG (polyethylene glycol $)^{78}$ and $\mathrm{F} 68^{111}$ to enable an extended circulation within the human body with potent pharmacological activities. TNIIA PEGylated nanoparticles (PEG-TNIIA-NPs) were prepared using the double emulsion/solvent evaporation technique and then conjugated with CBSA through the maleimide function, finally forming CBSA-PEG-TNIIA-NPs with a long circulating time of 7.89 hours and better uptake efficiency in the brain than a TNIIA solution. The study also indicated that CBSA-PEG-TNIIA-NPs, which exhibited significant neuroprotective effects in ischemic stroke, could suppress microglial activation, inhibit neutrophil infiltration, and downregulate multiple pro-inflammatory cytokines via the regulation of $\mathrm{p} 38 \mathrm{MAPK}$ and PPAR signaling pathways. ${ }^{78}$ Moreover, solid lipid NPs of CPT were prepared by an ultrasonic and high-pressure homogenization method to improve the low bioavailability with soy lecithin and Tween 80 as emulsifiers. ${ }^{83}$ Although TNIIA NPs have greater bioavailability as well as water solubility and a long circulation time in the human body with excellent pharmacological effects, it would be promising to prepare polymeric NPs of TNIIA with the capability of targeted delivery, which has attracted much attention due to the enhanced cell uptake of drugs with minimal systemic side effects. ${ }^{112}$ 


\section{Others}

In addition to the formulations of TNIIA mentioned earlier, pellets $^{113}$ and micelles ${ }^{114}$ have also been used to enhance the solubility and stability of TNIIA. Several materials were applied to the TNIIA pellets with sustained release, including hydroxypropyl methylcellulose (HPMC) and surelease, ${ }^{113}$ PVP and F68, ${ }^{115}$ polyvinyl acetate (PVAc), and poly(vinyl alcohol)poly(ethylene glycol) (PVA-PEG) graft copolymer. ${ }^{116}$ In addition, D-alpha-tocopheryl polyethylene glycol succinategraft-PLGA (TPGS-g-PLGA) copolymer and F68 were used to form mixed micelles with improved bioavailability and prolonged circulation times against hepatocellular carcinoma. ${ }^{114}$ However, neither of these formulations had a targeted delivery system for the improved cellular uptake of TNIIA.

\section{Conclusion}

To overcome the low bioavailability of tanshinones, many methods have been used to improve the solubility and dissolution rates as well as prolong the circulating times of these compounds; in particular, solid dispersion and NPs have shown better results than other formulations. In recent research, most of the formulations have had no tissue specificity. To enhance the pharmacological effects of TNIIA, it is necessary to design a type of drug preparation with components that actively target tumor tissue or other parts of the body. Despite the high dissolution rates of tanshinones obtained by these novel techniques, there has been little development in the clinical application of these formulations due to the complex process to produce the formulations and the lack of further research of TNIIA formulations on clinical trials, which is the ultimate goal in pharmaceutical research. In addition, it is necessary to clarify the light sensitivity of TNIIA in future research, and the development of TNIIA preparations may in part be driven by this lack of information. In conclusion, it is expected that with more and more effort for developing drug delivery systems of tanshinones and their clinical application, formulations of tanshinones can make a breakthrough and better therapy in the future.

\section{Acknowledgments}

This study was supported by the Macau Science and Technology Development Fund (102/2012/A3), the Research Fund of the University of Macau (MYRG2014-00033-ICMSQRCM, MYRG2014-00051-ICMS-QRCM, MRG005/ CMW/2014/ICMS), the National Natural Science Foundation of China (grant numbers 31271444 and 81201726), and the Guangdong Natural Science Funds for Distinguished Young Scholar (grant number 2014A030306001).

\section{Disclosure}

The authors report no conflicts of interest in this work.

\section{References}

1. Zhou L, Zuo Z, Chow MS. Danshen: an overview of its chemistry, pharmacology, pharmacokinetics, and clinical use. J Clin Pharmacol. 2005;45(12):1345-1359.

2. Wang XH, Morris-Natschke SL, Lee KH. New developments in the chemistry and biology of the bioactive constituents of tanshen. Med Res Rev. 2007;27(1):133-148.

3. Tse AK, Chow KY, Cao HH, et al. The herbal compound cryptotanshinone restores sensitivity in cancer cells that are resistant to the tumor necrosis factor-related apoptosis-inducing ligand. $J$ Biol Chem. 2013;288(41):29923-29933.

4. Cheng TO. Cardiovascular effects of Danshen. Int J Cardiol. 2007; 121(1):9-22.

5. Xu S, Liu P. Tanshinone II-A: new perspectives for old remedies. Expert Opin Ther Pat. 2013;23(2):149-153.

6. Park JH, Park Ok, Cho JH, et al. Anti-inflammatory effect of tanshinone I in neuroprotection against cerebral ischemia-reperfusion injury in the gerbil hippocampus. Neurochem Res. 2014;39(7):1300-1312.

7. Su CC, Chen GW, Lin JG. Growth inhibition and apoptosis induction by tanshinone I in human colon cancer colo 205 cells. Int J Mol Med. 2008;22(5):613-618.

8. Kim DH, Kim S, Jeon SJ, et al. Tanshinone I enhances learning and memory, and ameliorates memory impairment in mice via the extracellular signal-regulated kinase signalling pathway. Br J Pharmacol. 2009;158(4):1131-1142.

9. Tian XH, Wu JH. Tanshinone derivatives: a patent review (January 2006-September 2012). Expert Opin Ther Pat. 2013;23(1):19-29.

10. Gao S, Liu Z, Li H, Little PJ, Liu P, Xu S. Cardiovascular actions and therapeutic potential of tanshinone IIA. Atherosclerosis. 2012;220(1): $3-10$.

11. Wang JW, Wu JY. Tanshinone biosynthesis in Salvia miltiorrhiza and production in plant tissue cultures. Appl Microbiol Biotechnol. 2010;88(2):437-449.

12. Wu WY, Wang YP. Pharmacological actions and therapeutic applications of Salvia miltiorrhiza depside salt and its active components. Acta Pharmacol Sin. 2012;33(9):1119-1130.

13. Zhou X, Chan K, Yeung JH. Herb-drug interactions with Danshen (Salvia miltiorrhiza): a review on the role of cytochrome P450 enzymes. Drug Metabol Drug Interact. 2012;27(1):9-18.

14. Ma P, Liu J, Zhang C, Liang Z. Regulation of water-soluble phenolic acid biosynthesis in Salvia miltiorrhiza Bunge. Appl Biochem Biotechnol. 2013;170(6):1253-1262.

15. Wu WY, Yan $\mathrm{H}$, Wang XB, et al. Sodium tanshinone IIA silate inhibits high glucose-induced vascular smooth muscle cell proliferation and migration through activation of AMP-activated protein kinase. PLoS One. 2014;9(4):e94957.

16. Shang Q, Xu H, Huang L. Tanshinone IIA: a promising natural cardioprotective agent. Evid Based Complement Alternat Med. 2012;2012: 716459.

17. Liu QY, Zhang ZH, Jin X, Jiang YR, Jia XB. Enhanced dissolution and oral bioavailability of tanshinone IIA base by solid dispersion system with lowmolecular-weight chitosan. J Pharm Pharmacol. 2013;65(6):839-846.

18. Zhang Y, Wei L, Sun D, et al. Tanshinone IIA pretreatment protects myocardium against ischaemia/reperfusion injury through the phosphatidylinositol 3-kinase/Akt-dependent pathway in diabetic rats Diabetes Obes Metab. 2010;12(4):316-322.

19. Yuan X, Jing S, Wu L, Chen L, Fang J. Pharmacological postconditioning with tanshinone IIA attenuates myocardial ischemia-reperfusion injury in rats by activating the phosphatidylinositol 3-kinase pathway. Exp Ther Med. 2014;8(3):973-977.

20. Liu Z, Wang J, Huang E, et al. Tanshinone IIA suppresses cholesterol accumulation in human macrophages: role of heme oxygenase-1. J Lipid Res. 2014;55(2):201-213 
21. Xu S, Liu Z, Huang Y, et al. Tanshinone II-A inhibits oxidized LDLinduced LOX-1 expression in macrophages by reducing intracellular superoxide radical generation and NF-kappaB activation. Trans Res. 2012;160(2):114-124.

22. Chen W, Tang F, Xie B, Chen S, Huang H, Liu P. Amelioration of atherosclerosis by tanshinone IIA in hyperlipidemic rabbits through attenuation of oxidative stress. Eur J Pharmacol. 2012;674(2-3): 359-364.

23. Jin UH, Suh SJ, Chang HW, et al. Tanshinone IIA from Salvia miltiorrhiza BUNGE inhibits human aortic smooth muscle cell migration and MMP-9 activity through AKT signaling pathway. J Cell Biochem. 2008;104(1):15-26.

24. Wang J, Dong MQ, Liu ML, et al. Tanshinone IIA modulates pulmonary vascular response to agonist and hypoxia primarily via inhibiting $\mathrm{Ca} 2+$ influx and release in normal and hypoxic pulmonary hypertension rats. Eur J Pharmacol. 2010;640(1-3):129-138.

25. Luo Y, Xu DQ, Dong HY, et al. Tanshinone IIA inhibits hypoxiainduced pulmonary artery smooth muscle cell proliferation via Akt/ Skp2/p27-associated pathway. PLoS One. 2013;8(2):e56774.

26. Zheng L, Liu M, Wei M, et al. Tanshinone IIA attenuates hypoxic pulmonary hypertension via modulating K currents. Respir Physiol Neurobiol. 2014;205:120-128.

27. Liu L, Zhang X, Wang L, et al. The neuroprotective effects of tanshinone IIA are associated with induced nuclear translocation of TORC1 and upregulated expression of TORC1, pCREB and BDNF in the acute stage of ischemic stroke. Brain Res Bull. 2010;82(3-4):228-233.

28. Lee JC, Park JH, Park OK, et al. Neuroprotective effects of tanshinone I from Danshen extract in a mouse model of hypoxia-ischemia. Anat Cell Biol. 2013;46(3):183-190.

29. Lam BY, Lo AC, Sun X, Luo HW, Chung SK, Sucher NJ. Neuroprotective effects of tanshinones in transient focal cerebral ischemia in mice. Phytomedicine. 2003;10(4):286-291.

30. Yan FF, Liu YF, Liu Y, Zhao YX. Sulfotanshinone sodium injection could decrease fibrinogen level and improve clinical outcomes in patients with unstable angina pectoris. Int J Cardiol. 2009;135(2):254-255.

31. Adams JD, Wang R, Yang J, Lien EJ. Preclinical and clinical examinations of Salvia miltiorrhiza and its tanshinones in ischemic conditions. Chin Med. 2006;1:3.

32. Yuan SL, Wang XJ, Wei YQ. 丹參酮抗腫瘤作用及其機理的研 究 [Anticancer effect of tanshinone and its mechanisms]. Ai Zheng. 2003;22(12):1363-1366. Chinese.

33. Yun SM, Jung JH, Jeong SJ, Sohn EJ, Kim B, Kim SH. Tanshinone IIA induces autophagic cell death via activation of AMPK and ERK and inhibition of mTOR and $\mathrm{p} 70 \mathrm{~S} 6 \mathrm{~K}$ in KBM-5 leukemia cells. Phytother Res. 2014;28(3):458-464.

34. Zhang Y, Wei RX, Zhu XB, Cai L, Jin W, Hu H. Tanshinone IIA induces apoptosis and inhibits the proliferation, migration, and invasion of the osteosarcoma MG-63 cell line in vitro. Anticancer Drugs. 2012; 23(2):212-219.

35. Tsai MY, Yang RC, Wu HT, Pang JH, Huang ST. Anti-angiogenic effect of tanshinone IIA involves inhibition of matrix invasion and modification of MMP-2/TIMP-2 secretion in vascular endothelial cells. Cancer Lett. 2011;310(2):198-206.

36. Yuxian X, Feng T, Ren L, Zhengcai L. Tanshinone II-A inhibits invasion and metastasis of human hepatocellular carcinoma cells in vitro and in vivo. Tumori. 2009;95(6):789-795.

37. Shan YF, Shen X, Xie YK, et al. Inhibitory effects of tanshinone II-A on invasion and metastasis of human colon carcinoma cells. Acta Pharmacol Sin. 2009;30(11):1537-1542.

38. Nizamutdinova IT, Lee GW, Lee JS, et al. Tanshinone I suppresses growth and invasion of human breast cancer cells, MDA-MB-231, through regulation of adhesion molecules. Carcinogenesis. 2008;29(10): 1885-1892.

39. Zhang K, Li J, Meng W, Xing H, Yang Y. C/EBPbeta and CHOP participate in tanshinone IIA-induced differentiation and apoptosis of acute promyelocytic leukemia cells in vitro. Int J Hematol. 2010;92(4): $571-578$.
40. Wang J, Wang X, Jiang S, et al. Growth inhibition and induction of apoptosis and differentiation of tanshinone IIA in human glioma cells. J Neurooncol. 2007;82(1):11-21.

41. Liang Y, Yang Y, Yuan S, et al. 丹參酮IIA誘導原代培養人急性早 幼粒細胞白血病細胞分化 [Terminal differentiation of human acute promyelocytic leukemia (APL) cells induced by tanshinone II A in primary culture]. Ниа Xi Yi Ке Da Хие Хие Вао. 2000;31(2):207-210. Chinese.

42. Chiu SC, Huang SY, Chen SP, Su CC, Chiu TL, Pang CY. Tanshinone IIA inhibits human prostate cancer cells growth by induction of endoplasmic reticulum stress in vitro and in vivo. Prostate Cancer Prostatic Dis. 2013;16(4):315-322.

43. Gong Y, Li Y, Lu Y, et al. Bioactive tanshinones in Salvia miltiorrhiza inhibit the growth of prostate cancer cells in vitro and in mice. Int $J$ Cancer. 2011;129(5):1042-1052.

44. Hou LL, Xu QJ, Hu GQ, Xie SQ. 丹參酮IIA增強順鉑抗前列腺癌作 用及分子機制研究 [Synergistic antitumor effects of tanshinone II A in combination with cisplatin via apoptosis in the prostate cancer cells]. Yао Хие Хие Вао. 2013;48(5):675-679. Chinese.

45. Park IJ, Kim MJ, Park OJ, et al. Cryptotanshinone sensitizes DU145 prostate cancer cells to Fas(APO1/CD95)-mediated apoptosis through Bcl-2 and MAPK regulation. Cancer Lett. 2010;298(1):88-98.

46. Zhang G, Yang YM, Meng WT, Zhou J. 丹參酮IIA與三氧化二砷協 同誘導急性早幼粒白血病細胞调亡的研究 [Apoptosis of NB4 cells induced by tanshinone II A combined with arsenic trioxide]. Sichuan Da Хие Хие Bao Yi Xие Ban. 2010;41(1):57-61. Chinese.

47. Kan S, Cheung WM, Zhou Y, Ho WS. Enhancement of doxorubicin cytotoxicity by tanshinone IIA in HepG2 human hepatoma cells. Planta Med. 2014;80(1):70-76.

48. Su CC. Tanshinone IIA potentiates the efficacy of 5-FU in Colo205 colon cancer cells in vivo through downregulation of P-gp and LC3-II. Exp Ther Med. 2012;3(3):555-559.

49. Fang ZY, Lin R, Yuan BX, Yang GD, Liu Y, Zhang H. Tanshinone IIA downregulates the CD40 expression and decreases MMP-2 activity on atherosclerosis induced by high fatty diet in rabbit. J Ethnopharmacol. 2008;115(2):217-222.

50. Chen X, Zhou ZW, Xue CC, Li XX, Zhou SF. Role of P-glycoprotein in restricting the brain penetration of tanshinone IIA, a major active constituent from the root of Salvia miltiorrhiza Bunge, across the blood-brain barrier. Xenobiotica. 2007;37(6):635-678.

51. Hu T, To KK, Wang L, et al. Reversal of P-glycoprotein (P-gp) mediated multidrug resistance in colon cancer cells by cryptotanshinone and dihydrotanshinone of Salvia miltiorrhiza. Phytomedicine. 2014;21(11):1264-1272.

52. Yang YM, Liu T. 丹參酮IIA治療耐全反式維甲酸的急性早幼粒細 胞白血病1例報道 [Complete remission of acute promyelocytic leukemia resisting all-trans retinoic acid of one case treated by tanshinone II A]. Sichuan Da Xue Xue Bao Yi Xue Ban. 2006;37(6):965-967. Chinese.

53. Zhang Y, Jiang P, Ye M, Kim SH, Jiang C, Lu J. Tanshinones: sources, pharmacokinetics and anti-cancer activities. Int J Mol Sci. 2012;13(10):13621-13666.

54. Fan GW, Gao XM, Wang H, et al. The anti-inflammatory activities of tanshinone IIA, an active component of TCM, are mediated by estrogen receptor activation and inhibition of iNOS. J Steroid Biochem Mol Biol. 2009;113(3-5):275-280.

55. Jang SI, Kim HJ, Kim YJ, Jeong SI, You YO. Tanshinone IIA inhibits LPS-induced NF-kappaB activation in RAW 264.7 cells: possible involvement of the NIK-IKK, ERK1/2, p38 and JNK pathways. Eur J Pharmacol. 2006;542(1-3):1-7.

56. Zheng S, Ren Z, Zhang Y, Qiao Y. Anti-inflammatory mechanism research of tanshinone II A by module-based network analysis. Biomed Mater Eng. 2014;24(6):3815-3824.

57. Kim SY, Moon TC, Chang HW, Son KH, Kang SS, Kim HP. Effects of tanshinone I isolated from Salvia miltiorrhiza bunge on arachidonic acid metabolism and in vivo inflammatory responses. Phytother Res. 2002;16(7):616-620. 
58. Kang BY, Chung SW, Kim SH, Ryu SY, Kim TS. Inhibition of interleukin-12 and interferon-gamma production in immune cells by tanshinones from Salvia miltiorrhiza. Immunopharmacology. 2000;49(3): 355-361.

59. Cao EH, Liu XQ, Wang JJ, Xu NF. Effect of natural antioxidant tanshinone II-A on DNA damage by lipid peroxidation in liver cells. Free Radic Biol Med. 1996;20(6):801-806.

60. Lin R, Wang WR, Liu JT, Yang GD, Han CJ. Protective effect of tanshinone IIA on human umbilical vein endothelial cell injured by hydrogen peroxide and its mechanism. J Ethnopharmacol. 2006;108(2): 217-222.

61. Li YI, Elmer G, Leboeuf RC. Tanshinone IIA reduces macrophage death induced by hydrogen peroxide by upregulating glutathione peroxidase. Life Sci. 2008;83(15-16):557-562.

62. Tao S, Zheng Y, Lau A, et al. Tanshinone I activates the Nrf2-dependent antioxidant response and protects against As(III)-induced lung inflammation in vitro and in vivo. Antioxid Redox Signal. 2013;19(14) $1647-1661$.

63. Zhou S, Chen W, Su H, Zheng X. Protective properties of tanshinone I against oxidative DNA damage and cytotoxicity. Food Chem Toxicol. 2013;62:407-412.

64. Guo Y, Li Y, Xue L, et al. Salvia miltiorrhiza: an ancient Chinese herbal medicine as a source for anti-osteoporotic drugs. $J$ Ethnopharmacol. 2014;155(3):1401-1416.

65. Lee SY, Choi DY, Woo ER. Inhibition of osteoclast differentiation by tanshinones from the root of Salvia miltiorrhiza bunge. Arch Pharm Res. 2005;28(8):909-913.

66. Kwak HB, Yang D, Ha H, et al. Tanshinone IIA inhibits osteoclast differentiation through down-regulation of c-Fos and NFATc1. Exp Mol Med. 2006;38(3):256-264.

67. Kim HH, Kim JH, Kwak HB, et al. Inhibition of osteoclast differentiation and bone resorption by tanshinone IIA isolated from Salvia miltiorrhiza Bunge. Biochem Pharmacol. 2004;67(9):1647-1656.

68. Jung SH, Seol HJ, Jeon SJ, Son KH, Lee JR. Insulin-sensitizing activities of tanshinones, diterpene compounds of the root of Salvia miltiorrhiza Bunge. Phytomedicine. 2009;16(4):327-335.

69. Lee DS, Lee SH, Noh JG, Hong SD. Antibacterial activities of cryptotanshinone and dihydrotanshinone I from a medicinal herb, Salvia miltiorrhiza Bunge. Biosci Biotechnol Biochem. 1999;63(12) 2236-2239.

70. Ren Y, Houghton PJ, Hider RC, Howes MJ. Novel diterpenoid acetylcholinesterase inhibitors from Salvia miltiorrhiza. Planta Med. 2004; 70(3):201-204.

71. Wang X, Lee WY, Or PM, Yeung JH. Effects of major tanshinones isolated from Danshen (Salvia miltiorrhiza) on rat CYP1A2 expression and metabolism of model CYP1A2 probe substrates. Phytomedicine. 2009; 16(8):712-725.

72. Yu C, Ye S, Sun H, et al. PXR-mediated transcriptional activation of CYP3A4 by cryptotanshinone and tanshinone IIA. Chem Biol Interact. 2009;177(1):58-64.

73. Park JY, Kim JH, Kim YM, et al. Tanshinones as selective and slowbinding inhibitors for SARS-CoV cysteine proteases. Bioorg Med Chem 2012;20(19):5928-5935.

74. Zhang HS, Chen XY, Wu TC, Zhang FJ. Tanshinone II A inhibits tatinduced HIV-1 transactivation through redox-regulated AMPK/Nampt pathway. J Cell Physiol. 2014;229(9):1193-1201.

75. Yu XY, Lin SG, Zhou ZW, et al. Role of P-glycoprotein in the intestinal absorption of tanshinone IIA, a major active ingredient in the root of Salvia miltiorrhiza Bunge. Curr Drug Metab. 2007;8(4):325-340.

76. Fan LC, Teng HW, Shiau CW, et al. SHP-1 is a target of regorafenib in colorectal cancer. Oncotarget. 2014;5(15):6243-6251.

77. Wang L, Lai Y, Li C, Jiang X. Study on the intestinal absorption profiles of tanshinone IIA and its inclusion complex with cyclodextrin in rats. PDA J Pharm Sci Technol. 2009;63(5):390-400.

78. Liu X, An C, Jin P, Liu X, Wang L. Protective effects of cationic bovine serum albumin-conjugated PEGylated tanshinone IIA nanoparticles on cerebral ischemia. Biomaterials. 2013;34(3):817-830.
79. Ma H, Fan Q, Yu J, Xin J, Zhang C. Anticancer activities of tanshinone microemulsion against hepatocellular carcinoma in vitro and in vivo. Mol Med Rep. 2013;7(1):59-64.

80. Yan HM, Zhang ZH, Jiang YR, Ding DM, Sun E, Jia XB. An attempt to stabilize tanshinone IIA solid dispersion by the use of ternary systems with nano-CaCO3 and poloxamer 188. Pharmacogn Mag. 2014;10(suppl 2):S311-S317.

81. Yu H, Subedi RK, Nepal PR, Kim YG, Choi HK. Enhancement of solubility and dissolution rate of cryptotanshinone, tanshinone I and tanshinone IIA extracted from Salvia miltiorrhiza. Arch Pharm Res. 2012;35(8):1457-1464.

82. Zhang J, Huang M, Guan S, et al. A mechanistic study of the intestinal absorption of cryptotanshinone, the major active constituent of Salvia miltiorrhiza. J Pharmacol Exp Ther. 2006;317(3):1285-1294.

83. Hu L, Xing Q, Meng J, Shang C. Preparation and enhanced oral bioavailability of cryptotanshinone-loaded solid lipid nanoparticles. AAPS Pharm Sci Tech. 2010;11(2):582-587.

84. Malam Y, Loizidou M, Seifalian AM. Liposomes and nanoparticles: nanosized vehicles for drug delivery in cancer. Trends Pharmacol Sci. 2009;30(11):592-599.

85. Elbayoumi TA, Torchilin VP. Current trends in liposome research. Methods Mol Biol. 2010;605:1-27.

86. Iyer R. Review of surfactant evaluation methods and perturbations of components in phases to predict onset of emulsion instability. Particul Sci Technol. 2008;26(4):306-317.

87. Madene A, Jacquot M, Scher J, Desobry S. Flavour encapsulation and controlled release - a review. Int J Food Sci Technol. 2006;41(1): $1-21$.

88. Chu T, Zhang Q, Li H, et al. Development of intravenous lipid emulsion of tanshinone IIA and evaluation of its anti-hepatoma activity in vitro. Int J Pharm. 2012;424(1-2):76-88.

89. Ma H, Fan Q, Yu J, Xin J, Zhang C. Novel microemulsion of tanshinone IIA, isolated from Salvia miltiorrhiza Bunge, exerts anticancer activity through inducing apoptosis in hepatoma cells. Am J Chin Med. 2013;41(1):197-210.

90. Chang LC, Wu CL, Liu CW, Chuo WH, Li PC, Tsai TR. Preparation, characterization and cytotoxicity evaluation of tanshinone IIA nanoemulsions. J Biomed Nanotechnol. 2011;7(4):558-567.

91. McClements DJ. Nanoemulsion-based oral delivery systems for lipophilic bioactive components: nutraceuticals and pharmaceuticals. Ther Deliv. 2013;4(7):841-857.

92. Liang Z, Mao SJ, Yin ZN, Jin H, Li H, Chu T. 丹參酮IIA靜脈乳 劑的制備與質量評價 [Preparation and quality evaluation of intravenous tanshinone II (A) emulsion]. Zhongguo Zhong Yao Za Zhi. 2008;33(11):1249-1252. Chinese.

93. Solanki JN, Murthy ZVP. Controlled size silver nanoparticles synthesis with water-in-oil microemulsion method: a topical review. Ind Eng Chem Res. 2011;50(22):12311-12323.

94. Shakeel F, Ramadan W, Faisal MS, et al. Transdermal and topical delivery of anti-inflammatory agents using nanoemulsion/microemulsion: an updated review. Curr Nanosci. 2010;6(2):184-198.

95. Fan Q, Fan GJ, Yang PM, Zhao JY. 丹參酮微乳逆轉腫瘤細胞多藥耐 藥的研究 [Effect of tanshinone microemulsion on reversing MDR in human tumor cells]. Zhongguo Zhong Yao Za Zhi. 2004;29(11):10791081. Chinese.

96. Lv HY, Fan Q, Zhang N, Li BY. 丹參酮微乳制劑的HPLC指紋圖譜 研究 [Studies on HPLC fingerprints of tanshinone microemulsion]. Zhongguo Zhong Yao Za Zhi. 2004;29(11):1047-1049. Chinese.

97. Li HL, Zhang ZY, Ma LL, Chen XY. 丹參酮微乳的制備及大鼠在體腸 吸收 [Preparation of tanshinone microemulsion and its absorption in rat intestine in situ]. Zhongguo Zhong Yao Za Zhi. 2007;32(11):1024-1027. Chinese.

98. Laza-Knoerr AL, Gref R, Couvreur P. Cyclodextrins for drug delivery. J Drug Target. 2010;18(9):645-656.

99. Yuexian F, Junfen L, Chuan D. Preparation and study on the inclusion complexes of two tanshinone compounds with beta-cyclodextrin Spectrochim Acta A Mol Biomol Spectrosc. 2005;61(1-2):135-140. 
100. Ling W, Rui LC, Hua JX. In situ intestinal absorption behaviors of tanshinone IIA from its inclusion complex with hydroxypropyl-betacyclodextrin. Biol Pharm Bull. 2007;30(10):1918-1922.

101. Luo X, Xu YH, Chen B, Gu LQ, Huang M, Liu PQ. 羥丙基- $\beta$-環 糊精對隱丹參酮的增溶作用及其包合物的制備 [Solubilization on cryptotanshinone by hydroxypropyl-beta-cyclodextrin and preparation of their inclusion compound]. Zhongguo Zhong Yao Za Zhi. 2005;30(17):1328-1331. Chinese.

102. Zhao X, Liu X, Gan L, Zhou C, Mo J. Preparation and physicochemical characterizations of tanshinone IIA solid dispersion. Arch Pharm Res. 2011;34(6):949-959.

103. Yuan J, Mao S, Shen Q, Hou S, He Y. 載體聯用固體分散技術對丹 參酮IIA體外溶出的影響 [Influence of solid dispersion technique combination on dissolution of tanshinone IIA]. Zhongguo Zhong Yao Za Zhi. 2009;34(6):685-689. Chinese.

104. Jiang Y, Zhang Z, Lu Y, Tang J, Ma T, Jia X. 丹參酮IIA二元載體固 體分散體的研究 [Study on solid dispersion of binary vector of tanshinone II A]. Zhongguo Zhong Yao Za Zhi. 2012;37(10):1383-1387. Chinese.

105. Jiang YR, Zhang ZH, Liu QY, Hu SY, Chen XY, Jia XB. Preparation, characterization, and in vivo evaluation of tanshinone IIA solid dispersions with silica nanoparticles. Int J Nanomedicine. 2013;8:2285-2293.

106. Jiang YR, Zhang ZH, Xia HJ, Jia XB. 基于共聚維酮的丹參酮IIA 固體分散體的研究 [Study on solid dispersion of copovidone-based tanshinone II(A)]. Zhongguo Zhong Yao Za Zhi. 2013;38(2):174-178. Chinese.

107. Chow JC, Biswas P, Eatough D, et al. Nanoparticles and the environment - introduction. J Air Waste Manage. 2005;55(6):706-707.

108. Gan LC, Hou SX, Bi YQ, Wang CG, Wang XC, Chen QX. 2種方法 制備丹參酮IIA-PLGA納米粒的比較 [Comparison of two preparation methods applied in tanshinone II(A)-loaded PLGA nanoparticles]. Zhongguo Zhong Yao Za Zhi. 2007;32(7):578-581. Chinese.
109. Liang L, Chen Y, Xiong S, Zeng Z, Sun M, Zhang H. 丹參酮IIA納 米球對兔頸動脈球囊損傷后內膜增殖的抑制 [The inhibitive effect produced by local perfusion of tanshinone IIA nanoparticle on neointimal hyperplasia of rabbit carotid artery following intimal denudation]. Sheng Wu Yi Xue Gong Cheng Xue Za Zhi. 2007;24(4):812-816. Chinese.

110. Li Q, Wang Y, Feng N, Fan Z, Sun J, Nan Y. Novel polymeric nanoparticles containing tanshinone IIA for the treatment of hepatoma. J Drug Target. 2008;16(10):725-732.

111. Zhang WL, Liu JP, Liu XX, Chen ZQ. Stealth tanshinone IIA-loaded solid lipid nanoparticles: effects of poloxamer 188 coating on in vitro phagocytosis and in vivo pharmacokinetics in rats. Yao Xue Xue Bao. 2009;44(12):1421-1428.

112. Prabhu RH, Patravale VB, Joshi MD. Polymeric nanoparticles for targeted treatment in oncology: current insights. Int J Nanomedicine. 2015;10:1001-1018.

113. Liang XD, Zheng Y, Fan TY. 丹參酮IIA脈沖微丸的制備與體外質 量評價 [Preparation and in vitro evaluation of tanshinone IIA pulsatile release pellets]. Beijing Da Xue Xue Bao. 2010;42(5):559-564. Chinese.

114. Zhang J, Li Y, Fang X, Zhou D, Wang Y, Chen M. TPGS-g-PLGA/ pluronic F68 mixed micelles for tanshinone IIA delivery in cancer therapy. Int J Pharm. 2014;476(1-2):185-198.

115. Li J, Liu P, Liu JP, Zhang WL, Yang JK, Fan YQ. Novel tanshinone II A ternary solid dispersion pellets prepared by a single-step technique: in vitro and in vivo evaluation. Eur J Pharm Biopharm. 2012; 80(2):426-432.

116. Liu P, Li J, Liu J, Yang J, Fan Y. Release behavior of tanshinone IIA sustained-release pellets based on crack formation theory. J Pharm Sci. 2012;101(8):2811-2820.
International Journal of Nanomedicine

\section{Publish your work in this journal}

The International Journal of Nanomedicine is an international, peerreviewed journal focusing on the application of nanotechnology in diagnostics, therapeutics, and drug delivery systems throughout the biomedical field. This journal is indexed on PubMed Central, MedLine, CAS, SciSearch ${ }^{\circledR}$, Current Contents ${ }^{\circledR} /$ Clinical Medicine,

\section{Dovepress}

Journal Citation Reports/Science Edition, EMBase, Scopus and the Elsevier Bibliographic databases. The manuscript management system is completely online and includes a very quick and fair peer-review system, which is all easy to use. Visit http://www.dovepress.com/ testimonials.php to read real quotes from published authors. 